\title{
New basal cell carcinoma susceptibility loci
}

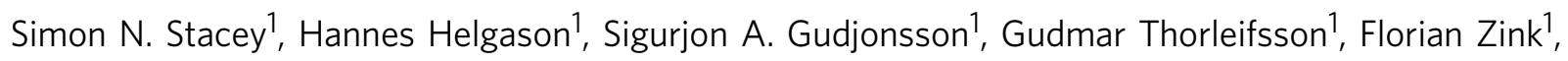
Asgeir Sigurdsson1, Birte Kehr1, Julius Gudmundsson1, Patrick Sulem', Bardur Sigurgeirsson 2,3, Kristrun R. Benediktsdottir 2,3, Kristin Thorisdottir ${ }^{2,3}$, Rafn Ragnarsson 2,3, Victoria Fuentelsaz ${ }^{4}$, Cristina Corredera ${ }^{5}$, Yolanda Gilaberte ${ }^{6}$, Matilde Grasa7, Dolores Planelles ${ }^{8}$, Onofre Sanmartin ${ }^{9,10}$, Peter Rudnai ${ }^{11}$, Eugene Gurzau ${ }^{12}$, Kvetoslava Koppova ${ }^{13}$, Bjørn A. Nexø ${ }^{14}$, Anne Tjønneland ${ }^{15}$, Kim Overvad ${ }^{16}$, Jon G. Jonasson 2,3,17, Laufey Tryggvadottir ${ }^{3,17}$, Hrefna Johannsdottir ${ }^{1}$, Anna M. Kristinsdottir ${ }^{1}$, Hreinn Stefansson¹, Gisli Masson¹, Olafur T. Magnusson¹, Bjarni V. Halldorsson 1,18, Augustine Kong ${ }^{1}$, Thorunn Rafnar1, Unnur Thorsteinsdottir 1,3, Ulla Vogel ${ }^{19}$, Rajiv Kumar ${ }^{20}$, Eduardo Nagore ${ }^{9,10}$, José I. Mayordomo 21, Daniel F. Gudbjartsson', Jon H. Olafsson ${ }^{2,3}$ \& Kari Stefansson 1,3

In an ongoing screen for DNA sequence variants that confer risk of cutaneous basal cell carcinoma (BCC), we conduct a genome-wide association study (GWAS) of 24,988,228 SNPs and small indels detected through whole-genome sequencing of 2,636 Icelanders and imputed into 4,572 BCC patients and 266,358 controls. Here we show the discovery of four new BCC susceptibility loci: 2p24 MYCN ( $r 557244888[C], O R=0.76, P=4.7 \times 10^{-12}$ ), 2q33 CASP8-ALS2CR12 (rs13014235[C], OR=1.15, $\left.\quad P=1.5 \times 10^{-9}\right), \quad 8 \mathrm{q} 21 \quad$ ZFHX4 (rs28727938[G], OR=0.70, $P=3.5 \times 10^{-12}$ ) and 10p14 GATA3 (rs73635312[A], OR =0.74, $\left.P=2.4 \times 10^{-16}\right)$. Fine mapping reveals that two variants correlated with rs73635312[A] occur in conserved binding sites for the GATA3 transcription factor. In addition, expression microarrays and RNA-seq show that rs13014235[C] and a related SNP rs700635[C] are associated with expression of CASP8 splice variants in which sequences from intron 8 are retained.

\footnotetext{
${ }^{1}$ deCODE Genetics/AMGEN, Sturlugata 8, Reykjavik 101, Iceland. ${ }^{2}$ Landspitali-University Hospital, Reykjavik 101, Iceland. ${ }^{3}$ Faculty of Medicine, University of Iceland, Reykjavik 101, Iceland. ${ }^{4}$ Division of Dermatology, Gregorio Marañón Hospital, Madrid 28007, Spain. ${ }^{5}$ Division of Dermatology, Quiron Hospital, 50009 Zaragoza, Spain. ${ }^{6}$ Division of Dermatology, San Jorge General Hospital, Huesca 22004, Spain. ${ }^{7}$ Division of Dermatology, University Hospital, Zaragoza 50009, Spain. ${ }^{8}$ Laboratory of Histocompatibility-Molecular Biology, Centro de Transfusión de la Comunidad Valenciana, Avenida del Cid, 65-A, Valencia 46014, Spain. ${ }^{9}$ Department of Oncology, Instituto Valenciano de Oncologia, Valencia 46009, Spain. ${ }^{10}$ Universidad Católica de Valencia, Valencia 46003, Spain. ${ }^{11}$ Department of Environmental Epidemiology, National Institute of Environmental Health, Budapest H-1450, Hungary. ${ }^{12}$ Health Department, Environmental Health Centre, Babes Bolyai University, Cluj, RO-Cluj-Napoca, Romania. ${ }^{13}$ Department of Environmental Health, Regional Authority of Public Health, Banska Bystrica SK-975 56, Slovakia. ${ }^{14}$ Department of Biomedicine, University of Aarhus, Aarhus C DK-8000, Denmark. ${ }^{15}$ Danish Cancer Society Research Centre, DK-2100 Copenhagen $\varnothing$, Denmark. ${ }^{16}$ Department of Public Health, Institute of Epidemiology and Social Medicine, University of Aarhus, Aarhus C DK-8000, Denmark. ${ }^{17}$ Icelandic Cancer Registry, Skogarhlid 8, Reykjavik 105, Iceland. ${ }^{18}$ Institute of Biomedical and Neural Engineering, School of Science and Engineering, Reykjavik University, Reykjavik 101, Iceland. ${ }^{19}$ National Research Centre for the Working Environment, Copenhagen DK-2100, Denmark. ${ }^{20}$ Division of Molecular Genetic Epidemiology, German Cancer Research Centre, Heidelberg D-69120, Germany. ${ }^{21}$ Division of Medical Oncology, University of Colorado, Aurora, Colorado 80045, USA. Correspondence and requests for materials should be addressed to S.N.S. (email: simon.stacey@decode.is) or to K.S. (email: kari.stefansson@decode.is).
} 
B asal cell carcinoma (BCC) is the most common cancer type in populations of European ancestry and its incidence has been increasing. Although it rarely metastasizes, it can be locally invasive and can cause considerable morbidity. Forty to fifty per cent of affected individuals develop new primary lesions within five years. The economic burden of treating and monitoring BCC is substantial ${ }^{1,2}$. In common with other forms of skin cancer, ultraviolet exposure is a major risk factor ${ }^{3}$.

Genetics plays a substantial role in BCC. Rare, high-penetrance inherited mutations in the Hedgehog pathway genes PTCH1, PTCH2 and SUFU cause Gorlin Syndrome (also called basal cell nevus syndrome $)^{1,4,5}$. Somatic mutations in Hedgehog pathway genes, in addition to TP53, are commonly observed in BCCs ${ }^{1,6}$. Genome-wide association and candidate gene studies have yielded a substantial number of low-to-moderate penetrance sequence variants that predispose to BCC. Some of the genes implicated, such as MC1R, ASIP, TYR, SLC45A2, OCA2 and IRF4 also affect pigmentation in Europeans ${ }^{7-13}$. Their effects on BCC risk are thought to be mediated at least in part by their influence on responses to ultraviolet exposure. Other genes implicated in BCC predisposition include PADI6/RCC2, RHOU, TERT, KRT5, CDKN2A/B, KLF14, TP53, TGM3 and RGS22, which have no obvious relation to ultraviolet-sensitive pigmentation traits ${ }^{10,14-17}$.

The Icelandic Cancer Registry has recorded histopathologically confirmed diagnoses of BCC since 1981, making studies using population-based case ascertainment possible ${ }^{18}$. We are conducting a search for BCC susceptibility loci based on variants discovered through whole-genome sequencing in the Icelandic population ${ }^{15}$. We use long-range phasing and imputation to determine the genotypes of these variants for single-nucleotide polymorphism (SNP) microarray chip-typed individuals and their relatives ${ }^{15,19,20}$. We now report that we have sequenced the whole genomes of 2,636 Icelanders to a median depth of $20 \times$. Genotypes of the variants detected by sequencing were imputed into 4,572 individuals with BCC (cases) and 266,358 controls using an algorithm that has been improved since our previous study ${ }^{16,21}$. Association analysis and replication genotyping in 1,475 cases and 4,733 control samples from nonIcelandic populations revealed evidence for four new BCC susceptibility loci.

\section{Results}

Loci associated with BCC. Imputation of genotypes for the variants identified by whole-genome sequencing allowed us to perform 24,988,228 tests of association; 19,543,184 tests for SNPs and $5,445,044$ tests for small indels $(<60 \mathrm{bp})$, testing only alleles that could be imputed with an information value above 0.8 . Association testing was performed using logistic regression, treating disease status as the response and genotype counts as covariates. A Q-Q plot is shown in Supplementary Fig. 1. In addition to reconfirming known BCC susceptibility loci, we identified four new loci that displayed substantial association signals (Supplementary Fig. 2).

We observed BCC association signals at 2 p24 from a group of variants with minor allele frequencies around $10.2 \%$. The strongest signal came from rs57244888[C], with odds ratio $(\mathrm{OR})=0.77, P=1.4 \times 10^{-8}$ (Fig. 1a, Table 1). The peak is in the intergenic region between $M Y C N$ and FAM49A and is separated from both genes by regions of moderate recombination rates (Fig. 1a). We generated a single-track Centaurus assay for rs57244888 and genotyped it in replication samples from Spain, Denmark and eastern Europe. The association replicated significantly in the non-Icelandic samples $(\mathrm{OR}=0.74, P=6.9$ $\times 10^{-5}$ ) and there was no evidence of heterogeneity between the sample sets (Table 1). Combining the Icelandic and non-Icelandic data provided strong overall evidence of an association $\left(\mathrm{OR}=0.76, P=4.7 \times 10^{-12}\right)$.

At $2 \mathrm{q} 33$, there were signals from a group of variants with population minor allele frequencies of about $46 \%$, represented by rs13014235[C] $\left(\mathrm{OR}=1.15, P=1.6 \times 10^{-7}\right.$, Fig. 1b). Significant replication was obtained in non-Icelandic sample sets $(\mathrm{OR}=1.15$, $P=0.0027)$ and combining all data sets yielded an $\mathrm{OR}=1.15$, $P=1.5 \times 10^{-9}$ (Table 1). rs13014235 is a p.Val34Leu missense variant in ALS2CR12 (amyotrophic lateral sclerosis 2 chromosomal region, candidate gene 12). The ALS2CR12 gene product is a structural component of the sperm flagellum ${ }^{22}$. Considered together with SIFT and PolyPhen predictions that the p.Val43Leu change is unlikely to have a functional consequence, ALS2CR12 seems a weak candidate for a BCC susceptibility gene.

Many variants are correlated with rs13014235 and they occur in a region of low recombination rate encompassing several genes (Fig. 1b). Two attractive candidate genes within the linkage disequilibrium block are CASP10 and CASP8. These genes encode initiator caspases of the extrinsic pathways of apoptosis, controlled by death receptors from the tumour necrosis factor receptor superfamily ${ }^{23}$. To highlight this, we designated the locus 2q33 CASP8-ALS2CR12.

We observed a cluster of signals at $8 \mathrm{q} 21$ from variant alleles with protective effects and frequencies around $6.2 \%$, typified by rs28727938 [G] $\left(\mathrm{OR}=0.70, P=2.1 \times 10^{-9}\right.$, Table 1, Fig. 1c). The signal peak is about $100 \mathrm{~kb}$ proximal to the zinc finger homeobox 4 ( $Z F H X 4)$ gene and close to a related antisense noncoding RNA ZFHX4-AS1. Genotyping of non-Icelandic samples showed significant replication $\left(\mathrm{OR}=0.69, P=4.0 \times 10^{-4}\right)$ and a combined Icelandic and non-Icelandic result of $\mathrm{OR}=0.70$, $P=3.5 \times 10^{-12}$ (Table 1$)$.

In a peak at $10 \mathrm{p} 14$, the strongest signal originated from rs73635312 with the minor [A] allele showing a protective effect $\left(\mathrm{OR}=0.73, P=2.3 \times 10^{-13}\right)$ and a population frequency of $12.6 \%$ (Fig. 1d, Table 1). The peak occurs in the region of the lincRNAs RP11-428L9.1 and RP11-428L9.2. The closest RefSeq gene to the peak is GATA3, although it is over $800 \mathrm{~kb}$ distal (Fig. 1d). The association was confirmed outside Iceland $\left(\mathrm{OR}=0.76, P=2.1 \times 10^{-4}\right)$. Combining the Icelandic and nonIcelandic data provided strong evidence of an association $\left(\mathrm{OR}=0.74, P=2.4 \times 10^{-16}\right.$, Table 1$)$.

To determine whether any independent signals exist at the loci, we carried out an association analysis of all variants in each region $\pm 500 \mathrm{~kb}$ surrounding each signal peak, conditioned on the effect of the top SNP. At 10p14 GATA3, we identified rs71483706 (Fig. 1d), whose minor allele conferred increased risk of BCC and had a frequency of about $32 \%(\mathrm{OR}=1.17, P=2.8$ $\left.\times 10^{-8}\right)$. In Iceland, rs71483706 retained a significant signal after adjustment for $r$ rs73635312 $\left(P_{\text {adj }}=4.2 \times 10^{-6}\right.$, $\left.\mathrm{OR}_{\mathrm{adj}}=1.14\right)$. However, the association could not be replicated in the non-Icelandic population samples $(P=0.31, \mathrm{OR}=1.06)$. There was no evidence of secondary signals at any of the other three loci.

Age at diagnosis. Sporadic BCC is predominantly a cancer of old age, with the majority of cases occurring in patients over the age of 60. However, in Gorlin syndrome, BCCs typically begin to appear in the early teens ${ }^{24}$. Accordingly, we investigated whether the variants we detected here and in earlier genome-wide association studies were associated with an earlier age at diagnosis of BCC (Supplementary Table 1). None of the four new variants reported here showed a significant association with age at diagnosis. The BCC variants reported previously had 
a

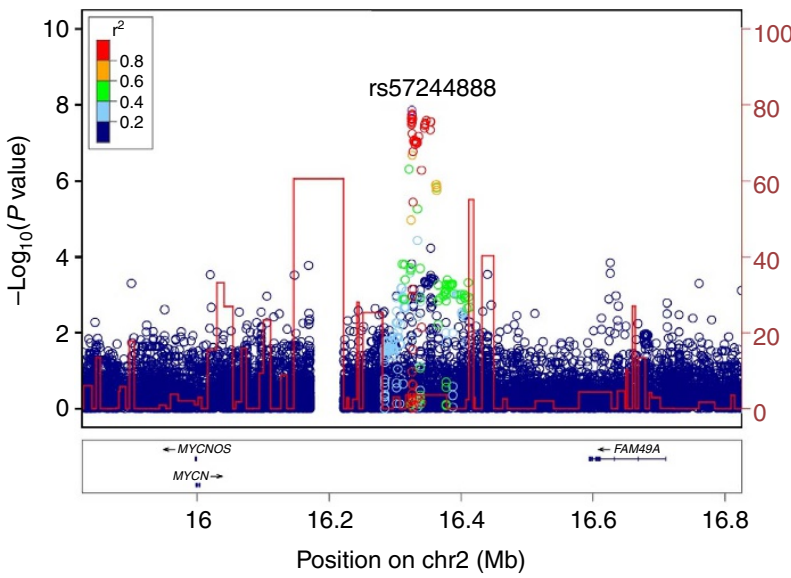

C

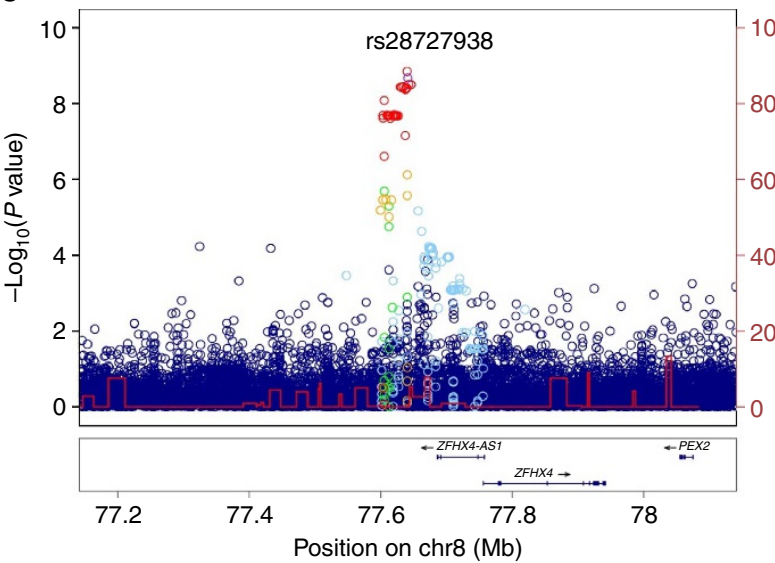

b

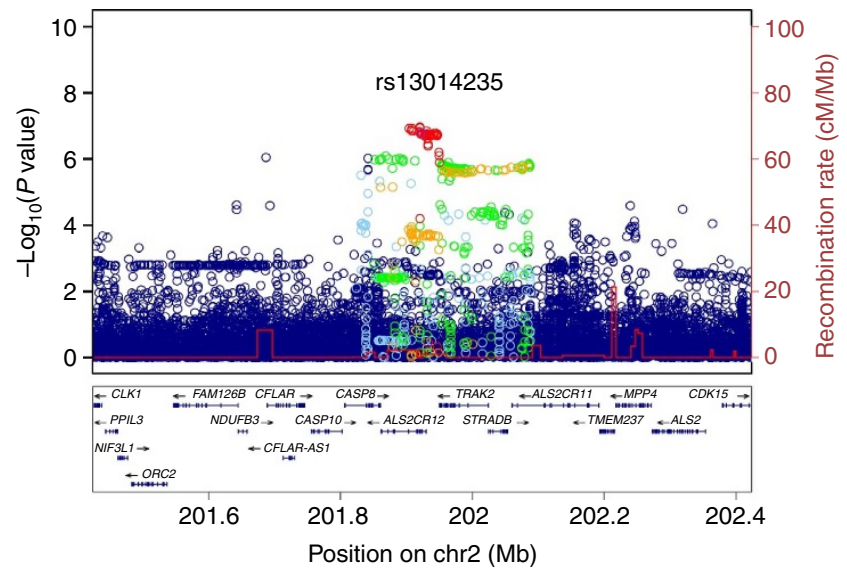

d

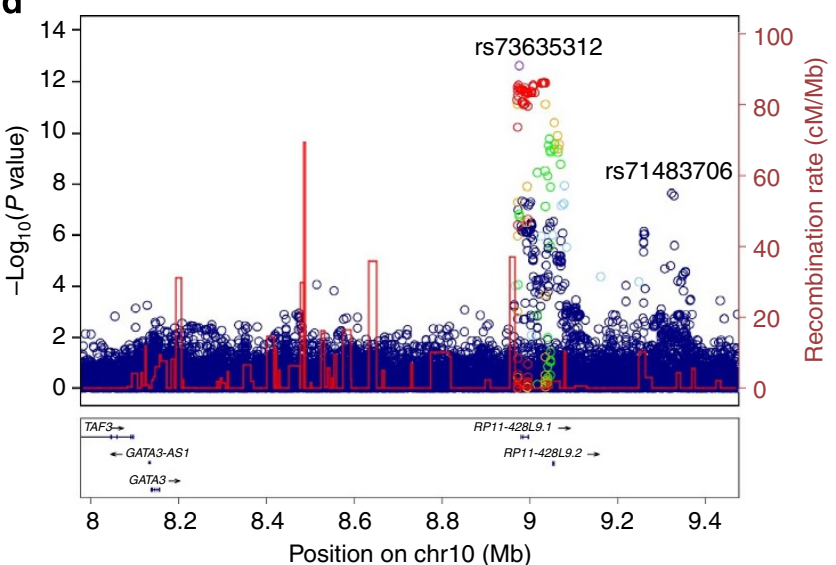

Figure 1 | New BCC association signals. Loci are (a) 2p24 MYCN, (b) 2q33 CASP8-ALS2CR12, (c) 8q21 ZFHX4 and (d) 10p14 GATA3 loci in the Icelandic sample. Data are based on association signals (expressed as $-\log _{10}(P)$ derived by logistic regression) for variants identified by whole-genome sequencing and imputation. The index SNP at each locus is labelled. Other variants are plotted in colours according to their $r^{2}$ values relative to the index SNP as indicated in the legend (a). Recombination rates, in $\mathrm{cM} / \mathrm{Mb}$ and based on Icelandic data, are plotted as red lines. The lower panel sections shows the locations of RefSeq genes and the chromosomal position (hg18 Build 36). The regions shown are $\pm 500 \mathrm{~kb}$ from the index SNP except for 10p14 GATA3, where the region is extended to $-1 \mathrm{Mb}$ to show the location of the GATA3 gene.

modest or no significant effects on age at diagnosis, though for the majority of variants the $\beta$ estimates were negative. Therefore, it appears that, unlike the high-penetrance variants associated with Gorlin syndrome, common BCC predisposing variants have little impact on age at diagnosis.

Fine mapping of variants to potentially functional sites. Because whole-genome sequencing was used for the detection and association testing of variants, we have a reasonably complete picture of the SNP and small indel variants present in Iceland down to a frequency of about $0.1 \%$. At each locus, we evaluated every variant's candidature for pathogenic effect using two main criteria: first, we searched for variants that are correlated with the index SNP and whose association with BCC was statistically indistinguishable from the index SNP. Second, we looked for co-localization of these variants with biologically relevant landmarks (see Methods). The results are presented in Supplementary Data 1.

For the 2p24 MYCN locus, one variant besides the top SNP was highlighted by this process. This SNP, rs73217623, is highly correlated with the top SNP rs57244888 $\left(r^{2}=1.0, P_{\text {adj }}=0.82\right)$. It is located in a DNaseI hypersensitivity site found in 54 cell types including normal human keratinocytes and in binding sites for seven transcription factors including CTCF in keratinocytes (Supplementary Data 1).

For the 2q33 CASP8-ALS2CR12 locus, 195 variants were indistinguishable from the index SNP rs13014235 with respect to BCC risk, of which 32 had biologically relevant annotations. Of note, rs2349075 is highly correlated with $\mathrm{rs} 13014235\left(r^{2}=0.99\right.$, $\left.P_{\text {adj }}=0.75\right)$ and is located near an enhancer, which is active in keratinocytes, amongst other cell types. Keratinocytes and 75 other cell types show evidence of DNaseI hypersensitivity at this location and DNaseI hypersensitivity is correlated with expression of CASP8. Ten transcription factors are known to bind at the site including members of the jun/fos family. The rs2349075 variant is predicted to alter an AP-1-binding site (Supplementary Data 1).

At the 8q21 ZFHX4 locus, two alleles of a multi-allelic indel that could not be distinguished from the top SNP rs28727938 had regulatory region annotations. However, neither they nor rs28727938 itself provided compelling evidence of a relevant function in keratinocytes (Supplementary Data 1).

For the 10p14 GATA3 locus, we noticed that two correlated variants, SNP rs17413266 and indel rs144203968 (both having $r^{2}=0.90$ versus rs73635312), occur in phylogenetically conserved regions which bind the GATA3 transcription factor. The SNP rs17413266 also has a Combined Annotation-Dependent 


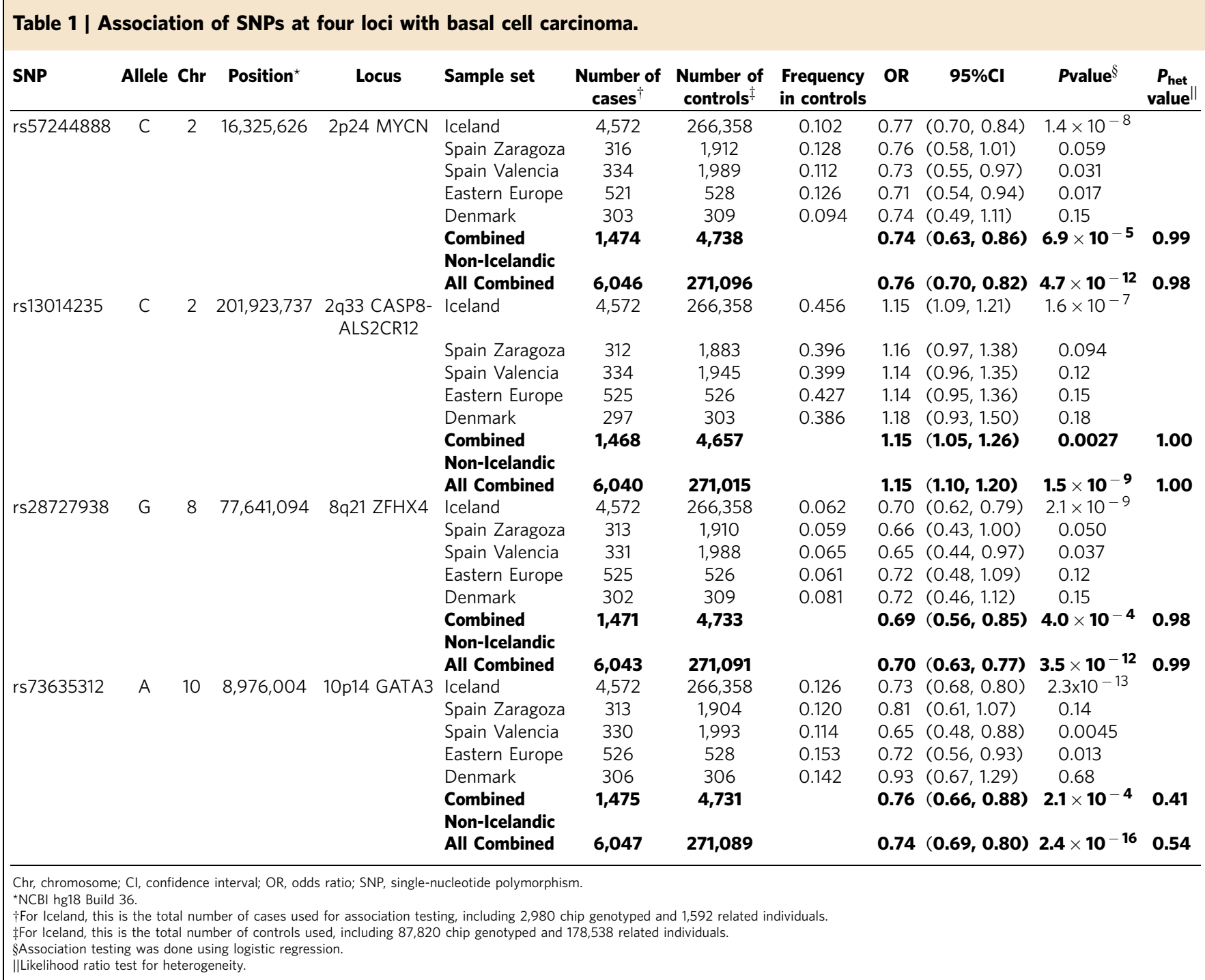

Depletion Scaled C-score of 20.3, which is in the 98.8th percentile for an intergenic variant ${ }^{25}$ (Supplementary Data 1).

Association with expression of CASP8 isoforms. We determined whether any of the BCC risk variants were associated with local differences in gene expression (cis-expression quantitative trait loci (eQTL)) by cross-referencing genotypes to RNA expression microarray data that we had derived previously from 1,001 blood and 673 adipose tissue samples ${ }^{26}$. No significant associations were seen for $2 \mathrm{p} 24 M Y C N, 8 \mathrm{q} 21$ ZFHX4 or $10 \mathrm{p} 14$ GATA3. At 2q33, CASP8 generates numerous splice variants (Fig. 2a). One probe on the microarrays, designated NM_033355, is in the $3^{\prime}$ UTR and captures all major CASP 8 isoform transcripts (Fig. 2c). A second probe, designated NM_033358, is unique to a small exon located between exons 8 and 9 of the major transcripts (exon numbering is based on NM_001228). The NM_033358 transcript encodes caspase- 8 isoform $\mathrm{E}$, which contains the death effector domains (DED) but lacks the catalytic domains of caspase-8 (Fig. 2b). A transcript with an extended exon 8, called exon 8L (NR_111983) has the capacity to encode a similar DEDonly isoform because the $8 \mathrm{~L}$ extension contains an in-frame stop codon. However, splicing of exon $8 \mathrm{~L}$ to downstream exons may target the transcript for nonsense-mediated decay ${ }^{27-30}$. Evidence for the existence in vivo of DED-only caspase-8 isoforms and their potential inhibitory effects on apoptosis has been controversial $^{28,29,31-34}$.

We found that the BCC risk allele rs13014235[C] is associated with a significant increase in abundance of transcripts containing the NM_033358 probe sequence in both blood $\left(P=1.9 \times 10^{-39}\right)$ and adipose tissue $\left(P=3.1 \times 10^{-19}\right)$. This is accompanied in adipose by a modest reduction in abundance of the major CASP8 transcripts as detected by probe NM_033355 (Supplementary Fig. 3). In fact, the expression of the isoform E transcript variant NM_033358 correlated negatively with expression of the major CASP8 transcript variants regardless of genotype $\left(P=2.1 \times 10^{-18}\right.$, Supplementary Fig. 4). A search of the surrounding genomic region identified rs700635[C], which is a member of the class of variants that could not be distinguished from rs13014235 with respect to BCC risk and which exhibited the strongest association with expression of the isoform E variant NM_033358 $(\beta=0.0698$, $P=1.2 \times 10^{-119}$ in blood RNA, Fig. 3). It also associated with a modest decrease in expression of the major CASP8 transcripts and had a similar pattern in adipose tissue.

Given the biological importance of CASP8, we initiated a more detailed investigation of its expression. We generated blood RNAseq data from an independent set of 261 genotyped individuals. For rs700635[C], we confirmed that RNA abundance in the 
a

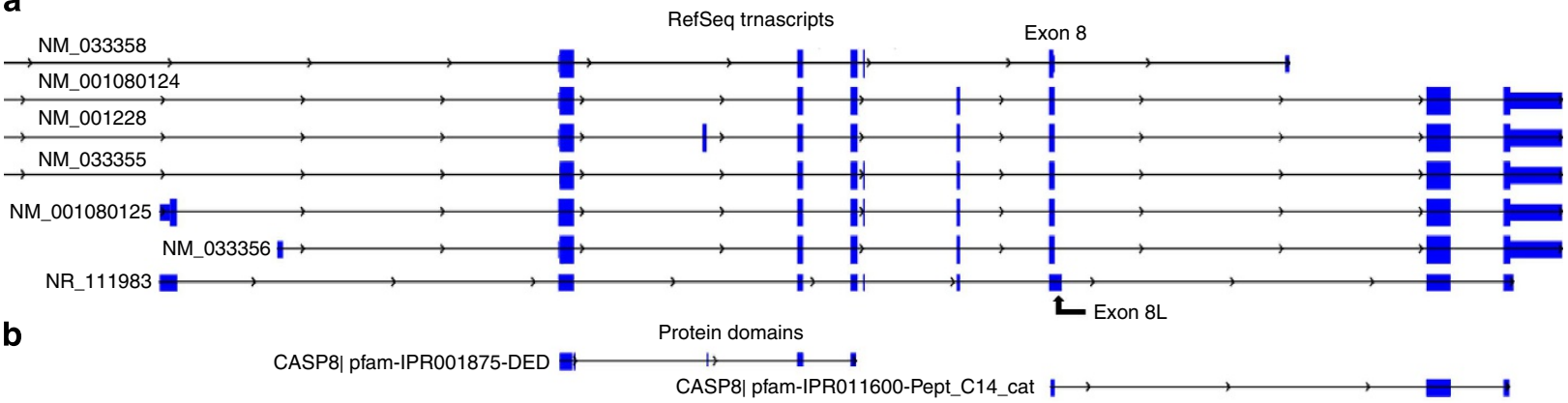

C

Expression microarray probes

RT-PCR probes

RT-PCR 8L splice variant

Figure 2 | CASP8-coding exons, protein domains and locations of probes. (a) Coding exons of the major transcript variants. The positions of exon 8 and exon $8 \mathrm{~L}$ are indicated. The transcript variant containing exon $8 \mathrm{~L}$ is designated 'NR_' because the exon $8 \mathrm{~L}$ sequence contains a stop codon that may render this RNA species susceptible to nonsense-mediated decay. (b) Procaspase-8 protein domains corresponding to exons, from pfam: IPR001875-DED are the DED; IPR011600-Pept_C14_cat corresponds to the peptidase catalytic domains. (c) Location of the probe sequences used on the expression microarrays. The height of the bars corresponds to the relative levels of expression in blood. (d) Location of primer-amplicons used in RT-PCR.

a

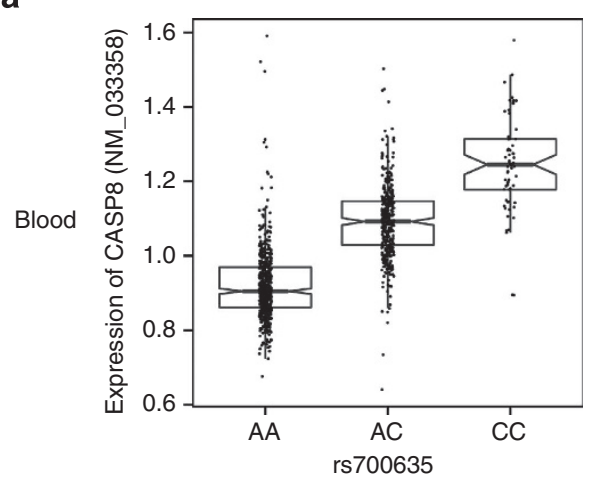

C

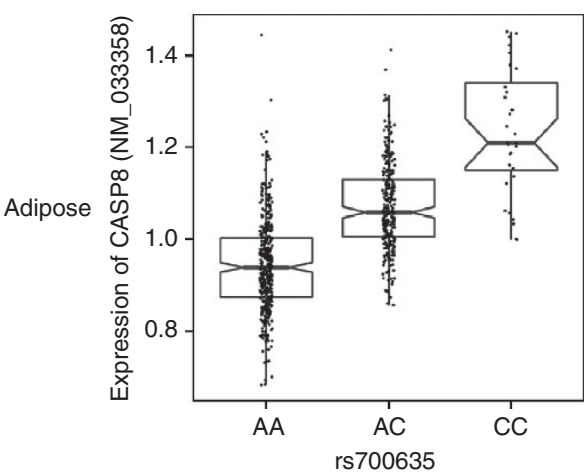

b

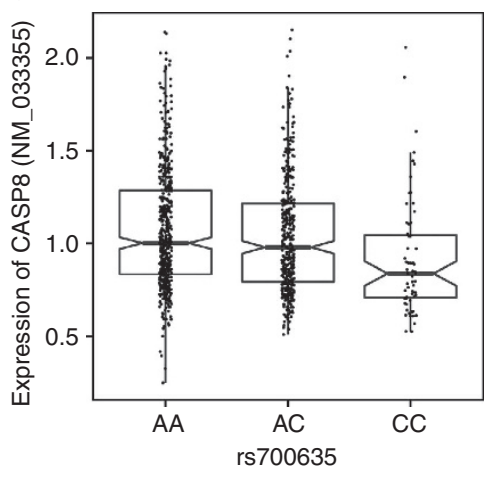

d

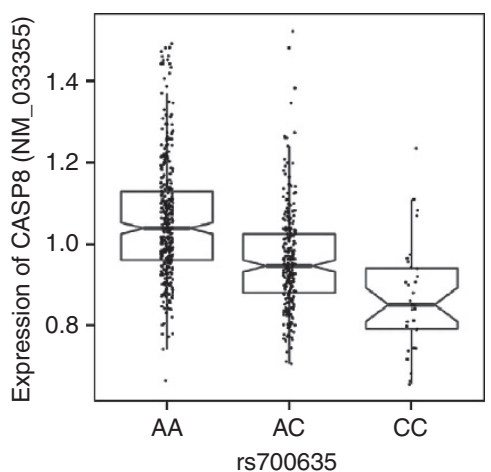

Figure 3 | RNA expression microarray data for CASP8 transcript variants, stratified by genotype of BCC risk variant rs700635. RNA from blood and adipose tissue was hybridized to Agilent expression microarrays containing probes for the major CASP8 transcript variants (NM_033355) and the variant encoding isoform E (NM_033358). Normalized expression levels were stratified by genotype of variant rs700635, allele [C] being the risk allele for BCC. Effects and $P$ values were determined using multivariable linear regression. The box plots show median, 25th and 75th percentiles (boxes) and 5th and 95th percentiles (vertical lines). Individual points for each sample are also plotted. (a) Expression of the isoform E transcript variant NM_033358 in blood. eQTL result coded to rs700635[C] is $\beta=0.0698, P=1.2 \times 10^{-119}$. (b) Expression of the major transcript variants NM_033355 in blood. $\beta=-0.0195$,

$P=0.0076$. (c) Expression of the isoform E transcript variant NM_033358 in adipose tissue. $\beta=0.0571 P=3.7 \times 10^{-63}$. (d) Expression of the major transcript variants NM_033355 in adipose tissue. $\beta=-0.0436, P=7.9 \times 10^{-22}$. 
NM_033358 probe region is increased in risk allele carriers. However, RNA-seq revealed that the entire intron 8 sequence between exon $8 \mathrm{~L}$ and the probe region is preferentially retained in carriers (Fig. 4a,b). This coincides with the reduced expression of the major exons of the CASP8 gene in association with rs700635[C] $\quad\left(\beta=-0.65 \quad P=1.7 \times 10^{-12}\right.$, Supplementary Table 2). Reverse transcription-PCR (RT-PCR) of 76 blood RNA samples (using probes as shown in Fig. 2d) reconfirmed these findings (Supplementary Fig. 5).

Retention of intron 8 appears to result from failures to recognize splice donors at the $3^{\prime}$ ends of exon 8 and exon $8 \mathrm{~L}$, with the exon $8 \mathrm{~L}$ donor being ignored preferentially in rs700635[C] carriers $\left(P=6.2 \times 10^{-22}\right.$, Supplementary Fig. 6). Some intron 8 reads had paired ends spliced from upstream exons, which helped to establish the sense strand polarity of the retained intron transcripts. We also saw reads indicating that exon 8 (but not exon 8L) can splice to the NM_033358 probe region. However, the efficiency of this splice did not vary by genotype $(P=0.89$, Supplementary Fig. 6). Of the 195 variants that were indistinguishable from the index SNP with respect to BCC risk, none occurred at the exon 8 or $8 \mathrm{~L}$ splice donor sites or the exon 9 splice acceptor. Limited data are available from intron 8 beyond the probe region, as there is very restricted read coverage $3^{\prime}$ to the NM_033358 probe in both RNA and DNA sequences. Nevertheless, we did detect RNA-seq read pairs crossing the intron 8exon 9 boundary, suggesting that the entirety of intron 8 fails to splice out in association with rs700635[C].

To investigate whether similar phenomena occur at CASP8 in epidermis, we obtained RNA-seq and genotype data from the GTEx project ${ }^{35}$ and analysed them using the same methods as described above (Supplementary Fig. 7). In sun-exposed skin, we again found that intron 8 including the NM_033358 probe sequence is expressed preferentially in association with the rs700635[C] BCC risk allele $\left(\beta=1.17, P=1.8 \times 10^{-24}\right.$ for intron 8 , Supplementary Table 2). The major CASP8 isoform variants were also expressed at reduced levels in BCC risk allele carriers in sun-exposed skin $\left(\beta=-0.64, P=7.2 \times 10^{-9}\right)$, in agreement with our findings in blood and adipose tissue. We did notice that preferential expression from rs700635[C] extended to sequences in exon 8 and intron 7, which was not seen by us in blood and remains unexplained (Supplementary Fig. 7). A search of the
GTEx sun-exposed skin RNA-seq data for eQTLs related to the lead SNPs at 2q24 MYCN, 8q21 ZFHX4 and 10p14 GATA3 yielded no significant associations.

Risk variants for other cancers at CASP8. CASP8 has been implicated in several cancer types both through germline variants and somatic mutations. The p.Asp302His germline variant rs1045485[C] has been associated with protection from breast cancer $^{36,37}$. We confirmed this association to nominal significance in the Icelandic population $(\mathrm{OR}=0.93, P=0.031)$. However, we saw no effect of the allele on BCC risk $(\mathrm{OR}=1.04, P=0.31,95 \%$ confidence interval $(\mathrm{CI})=0.97-1.11)$. CASP8 was also suggested as the likely candidate gene underlying the effect of a melanoma susceptibility variant in ALS2CR12 (rs13016963) (ref. 38). In our data, we could not see evidence of this melanoma association $(\mathrm{OR}=1.04, P=0.40,95 \% \mathrm{CI}=0.96-1.12)$. There was a nominal association of rs13016963 with $\mathrm{BCC}(\mathrm{OR}=1.08, P=0.0036)$ but it was fully accounted for by rs13014235, the index BCC SNP at the locus $\left(P_{\text {adj }}=0.79\right)$. A common chronic lymphocytic leukaemia risk variant ${ }^{39}$ did not show a BCC association ( $r 33769825, O R=1.03$, $P=0.35,95 \% \mathrm{CI}=0.97-1.08$ ), nor did the so-called $-6526 \mathrm{~N}$ del CASP8 promoter deletion (rs3834129) that has been controversially implicated in susceptibility to a variety of cancers $^{40,41} \quad(\mathrm{OR}=0.95, \quad P=0.069, \quad 95 \% \quad \mathrm{CI}=0.91-1.00)$. Moreover, we have not as yet detected a significant association between the BCC index SNP rs13014235 and any other type of cancer documented by the Icelandic Cancer Registry. Recently, Lin et al. $^{42}$ reported an association between rs1830298 in ALS2CR12 and breast cancer. That variant is highly correlated with the strongest CASP8 eQTL variant we report (rs700635, $\left.r^{2}=1.0\right)$. We confirmed the association with breast cancer for rs700635 ( $\mathrm{OR}=1.08, P=0.0053)$, but did not see a significant association with $\mathrm{rs13014235}(\mathrm{OR}=1.03, P=0.27)$. This suggests a complex pattern of cancer risk associations at the locus, reminiscent of the TERT-CPTLM1L locus previously identified in our BCC genomewide association study ${ }^{17}$.

\section{Discussion}

We report here on the discovery of four new susceptibility loci for BCC. At the $2 \mathrm{q} 24$ locus, $M Y C N$ is a known oncogene with a

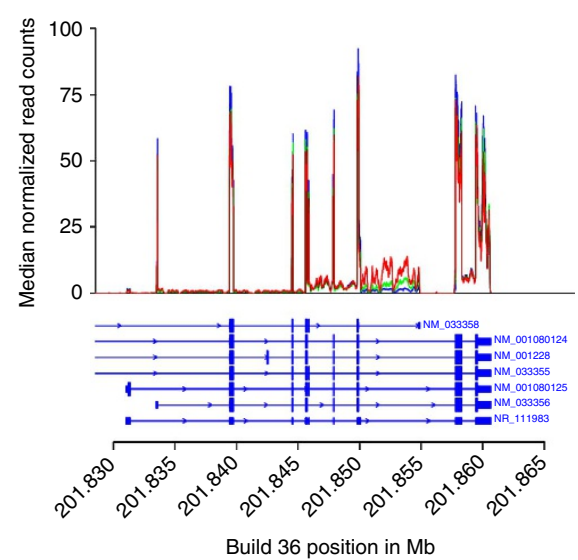

b

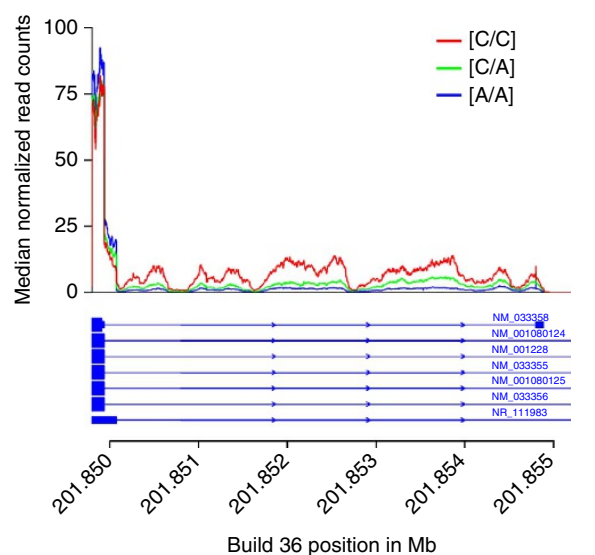

c

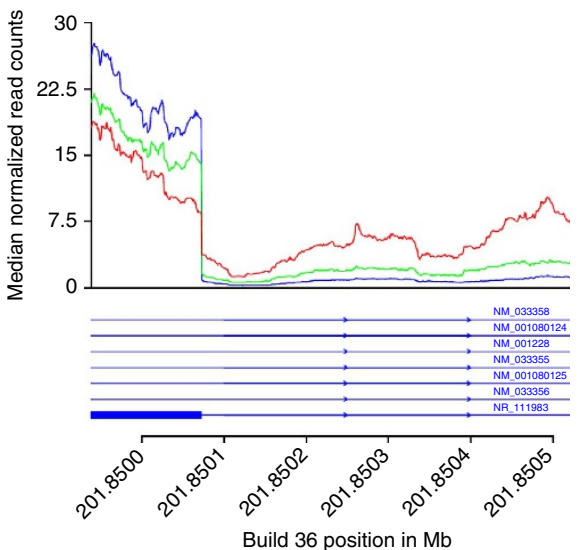

Figure 4 | Carriers of the rs700635[C] BCC risk allele show preferential retention of CASP8 intron 8. RNA-seq data was obtained from blood from genotyped donors. These were $n=24 \mathrm{rs} 700635[\mathrm{C} / \mathrm{C}]$ homozygotes (shown in red), $n=117 \mathrm{rs} 700635[\mathrm{C} / \mathrm{A}]$ heterozygotes (shown in green) and $n=114$ rs700635[A/A] homozygotes (shown in blue). The $x$ axis is the genomic position in Mb (hg18/Build36). The $y$ axis is, for each genotypic group, the median count of normalized reads (normalized for each individual to the total number of aligned reads). The structure of the RefSeq transcript variants is shown beneath the graphs. (a) The genomic region covering the coding exons of CASP8. (b) Zoom showing the exon 8 , exon $8 \mathrm{~L}$ region extending through intron 8 to the minor exon targeted by the NM_033358 probe. (c) Zoom showing the splice junction between exon $8 \mathrm{~L}$ and intron 8 . 
involvement in neuroblastoma and medulloblastoma ${ }^{43,44}$; however, it has not been implicated previously in BCC. Similarly, there are no reports of an involvement of ZFHX4 in cancer predisposition. CASP 8 and GATA3, on the other hand, do have known associations with both oncogenesis and epidermal development.

Following death receptor ligation, procaspase- 8 is recruited to the cytoplasmic death-inducing signalling complex (DISC) through interactions involving its DED. Recruitment of procaspase- 8 to the DISC leads to its dimerization and activation through induced proximity. Full activation of caspase- 8 results from cleavage of the catalytic domains from the DED and release of the dimerized catalytic domains from the DISC ${ }^{23}$. Our findings indicate that in BCC risk allele carriers there is a preferential retention of the intron following exons 8 and 8L. RNA species with this structure have the capacity to encode caspase- 8 isoforms, which contain the DED but lack the catalytic domains. Conceptually, an isoform with this structure has the potential to inhibit caspase- 8 signalling by interfering with the binding of full-length procaspase-8 to the DISC. Indeed some (but not all) investigators have observed anti-apoptotic effects resulting from the expression of DED-only caspase-8 isoforms $28,33,34$. If the RNA species run through to exon 9, splicing of exon 9 to exon 10 could, however, render the transcripts susceptible to nonsense-mediated decay. Coincident with the intron retention is a reduction in overall expression of the major transcript variants of CASP8. Combined, these factors could suppress caspase-8 signalling in risk allele carriers.

One predictable outcome of reduced caspase- 8 signalling might be a failure of incipient tumour cells to undergo apoptosis. During normal epidermal development there does not, however, seem to be an involvement of activated pro-apoptotic caspases ${ }^{45}$. Conditional knockout mice where CASP8 is specifically disabled in epidermal keratinocytes develop a lethal neonatal skin inflammation and hyperplasia ${ }^{46,47}$. Double knockout of CASP8 and the necroptosis-associated kinase RIPK3 rescues mice from neonatal lethality, skin inflammation and hyperplasia ${ }^{23,48}$. This suggests that CASP8 normally functions to suppress necroptosis and consequent inflammation in epidermis. It is therefore possible that an inhibition of caspase-8 signalling in BCC risk allele carriers could act to promote necrosis and inflammation, thereby generating a tumour-promoting environment within the epidermis.

We caution that while the effect of rs700635 on the expression of CASP8 is robustly proven and the SNP is associated with risk of $\mathrm{BCC}\left(\mathrm{OR}=1.15, P=1.0 \times 10^{-6}\right.$ in Iceland), rs700635 is not the most strongly associated BCC risk variant at the locus. Although rs700635 is included on the list of potentially causative variants, it is not extremely well correlated with the index SNP rs13014235 $\left(r^{2}=0.48\right)$ and rs13014235 retains a nominally significant BCC association signal when adjusted for the effect of rs700635 ( $P_{\mathrm{adj}}=0.022$, Supplementary Data 1$)$. The relationship between BCC risk and CASP8 transcript variant expression therefore remains tentative and merits further study. There are genes besides CASP8 at the 2q33 locus that should be considered. Like CASP8, CASP10 encodes an extrinsic apoptosis initiator caspase. It is less well characterized, in part, because it has no counterpart in mice. Somatic mutations in CASP10 have been found in gastric cancer and non-Hodgkin's lymphoma ${ }^{49,50}$. Also, in the region is $S T R A D B$, encoding a pseudokinase that complexes with STK11 and may have anti-apoptotic functions ${ }^{51}$.

The closest gene to the 10p14 signal is GATA3, encoding a $\mathrm{C} 2 \mathrm{H} 2$ zinc finger type transcription factor that binds to sequences resembling $\mathrm{A} / \mathrm{T}$ GATA $\mathrm{A} / \mathrm{G}$ in the proximity of target genes, where it can activate or repress transcription. GATA3 is involved in lineage specification and differentiation in a variety of settings, including $\mathrm{T}$ cells, mammary gland epithelia and $\operatorname{skin}^{52}$. In skin, GATA3 is involved in lineage determination of the inner root sheath of the hair follicle. GATA3-knockout mice and conditional knockouts, where GATA3 is specifically disrupted in the skin, show a failure of correct specification of inner root sheath cells and consequent disruption of hair follicle development ${ }^{53,54}$. In normal interfollicular skin, GATA3 is expressed in the suprabasal layer and persists throughout the differentiating layers ${ }^{54,55}$. GATA3 knockouts show a hyperplasia of the basal layer, disruption of the stratified epithelial differentiation programme and skin barrier defects ${ }^{53,54,56}$. Taken together with our association data these reports suggest that, in humans, variants at the GATA3 locus may affect the specification and differentiation programme of the epidermis.

Although GATA3 is the nearest gene to the variants we describe at 10p14, the signal peak is nevertheless a substantial distance $(>800 \mathrm{~kb})$ downstream of the gene. Analysis of ENCODE data revealed two highly correlated variants (rs17413266 and rs144203968) that occur in ChIP-Seq verified GATA3 binding sites. Ouyang et al. ${ }^{57}$ have shown that in CD4 ${ }^{+}$ T cells, GATA3 initiates an auto-activation feedback loop that promotes ongoing GATA3 expression and stabilizes Th2 lineage commitment. The molecular details of this auto-activation have never been worked out and it is not known if a similar feedback loop occurs in differentiating epidermis.

In summary, we have found four new BCC susceptibility loci. While the mechanisms at two of the loci remain cryptic, we provide testable hypotheses for roles of CASP 8 and GATA3 variants in epidermal carcinogenesis.

\section{Methods}

Subjects. Approval for the Icelandic study was granted by the Icelandic National Bioethics Committee and the Icelandic Data Protection Authority. All participants provided informed consent. Affected individuals were identified through the Icelandic Cancer Registry (ICR), which has maintained records of BCC diagnoses since 1981 . The records contained only cases of histologically verified BCC, sourced from all the pathology laboratories in the country that deal with these lesions. Icelandic controls consisted of individuals selected from other ongoing association studies at deCODE and who did not have a diagnosis of BCC recorded in the ICR Median age at diagnosis for ICR notified cases was 68 years (range 10-104). All subjects were of European ancestry. Compared with our most recent prior publication on $\mathrm{BCC}^{16}$, the present study adds 364 new BCC cases and 156,950 new controls. Of these, 254 cases and 16,944 controls were typed on Illumina chips resulting in a total of 17,198 new chip genotypes being available to the study. There was also an increase in the numbers of close relatives for whom genotype probabilities could be used: 110 additional BCC cases and 140,006 controls

Eastern European BCC cases were recruited from all general hospitals in three study areas in Hungary, two in Romania and one in Slovakia. Cases were identified based on histopathological examinations by pathologists. The median age at diagnosis was 67 years (range 30-85). Controls were recruited from the same hospitals from surgical, orthopaedic and trauma patients. Controls were thoroughly screened and individuals with past or current BCC, malignant disease, cardiovascular disease or diabetes were excluded. All subjects were of self-reported European ancestry. The study was approved by the Ethical Committee of the National Health Research Council, Hungary. In Romania, ethical aspects of the study were approved by Environmental Health Centre and Public Health

Department. In Slovakia, ethical aspects of the study were approved by the Ethical Committee of State Health Institutes.

Spain Valencia. Participants were recruited from the outpatient dermatology clinics of the Instituto Valenciano de Oncología in Valencia, Spain, starting from May 2003. Cases were patients with histologically proven BCC presenting with superficial or nodular lesions of $<1 \mathrm{~cm}$ in diameter. Clinical and pathological data from these patients are prospectively collected by medical history review, personal interview and clinical examination by an expert dermatologist.

Immunocompromised patients were excluded as were those with any autoimmune disease, hereditary disorders that include the presence of BCC (Gorlin syndrome, xeroderma pigmentosum) and epidermodysplasia verruciformis. Controls were disease free and ethnically matched healthy subjects recruited at the Transfusion Centre of Valencia. Phenotypic characteristics of controls were obtained by a selfadministered structured questionnaire. Prospective controls reporting past or present BCC were excluded. All subjects were of self-reported European ancestry All patients in the study had signed an informed consent and the study protocol was approved by the ethics boards of the Instituto Valenciano de Oncología and the Transfusion Centre of Valencia. 
Spain Zaragoza. BCC cases were recruited from the Oncology Department of Zaragoza University Hospital starting from September 2007. Individuals with histologically proven invasive BCC were eligible to participate in the study. The median time interval from BCC diagnosis to collection of blood samples was 14 months (range 1-53 months). Median age at diagnosis was 69 years (range 21-91). Controls were recruited from individuals who attended the Zaragoza University Hospital for diseases other than cancer. Prospective control subjects were questioned by the recruiter about past or present malignant disease, including BCC, and those with a positive history were excluded. All subjects were of selfreported European ancestry. All subjects in the study had signed an informed consent and the study protocol was approved by the Zaragoza University Hospital ethics board.

Denmark. Subjects were participating in the 'Diet, Cancer and Health' study, which is a prospective study of 57,053 individuals recruited at two centres in Denmark between December 1993 and May 1997 (ref. 58). Subjects were monitored during the follow-up period and BCC cases were identified through linkage to entries in the Danish Cancer Registry. Each case identified was matched to one control from the Diet, Cancer and Health cohort based on gender, age on study entry and age at diagnosis of the case. Median age at diagnosis was 59 (range 50-68). In addition to extensive diet and lifestyle questions, subjects answered questions regarding their skin sensitivity to sun, tanning during summer, presence of nevi and presence of freckles. All subjects were of self-reported European ancestry. The study 'Diet, Cancer and Health' has been approved by the regional Ethical Committees on Human Studies in Copenhagen and Aarhus, and by the Danish Data Protection Agency. Informed consent was obtained from all participants to search information from medical registers including the Danish Cancer Register.

Illumina SNP chip genotyping. The Icelandic chip-typed samples were assayed with the Illumina HumanHap300, HumanHapCNV370, HumanHap610, 1M, or Omni-Quad bead chips at the deCODE Genetics facility. All samples with a call rate of $<97 \%$ were removed from the analysis. SNPs were excluded if they had (i) a yield lower than $95 \%$, (ii) a minor allele frequency of $<0.01$ in the population, (iii) an excessive deviation from the Hardy-Weinberg equilibrium $\left(P<10^{-6}\right)$, (iv) an excessive inheritance error rate $(>0.001)$ or $(v)$ if there was a substantial difference in allele frequency between chip types (in which case the SNP was removed from a single chip type if that resolved the difference, but if it did not then the SNP was removed from all chip types). None of the SNPs listed in Table 1 showed a significant association with chip type ( $P$ values were between 0.11 and 0.77 , tested using logistic regression).

Whole-genome sequencing imputation and association testing. In this study, we sequenced 2,636 Icelanders to a median depth of $20 \times$. Long-range haplotype phasing and genealogy-based calculation of genotype probabilities ${ }^{15,19-21}$ of the variants identified by sequencing allowed us to perform 24,988,228 tests of association; 19,543,184 tests for SNPs and 5,445,044 tests for small indels ( $\leq 60 \mathrm{bp}$ ). We tested only alleles that could be imputed with an information value $\geq 0.8$, where the informativeness of genotype imputation was estimated by the ratio of the variance of imputed expected allele counts to the variance of the actual allele counts, as described previously ${ }^{15}$. This permitted us to test variants down to a minor allele frequency of $\sim 0.1 \%$. In association testing, we tested each allele against all other alleles at that position combined. Thus, for biallelic variants, this corresponds to one test, whereas for multi-allelic variants this corresponds to $n$ tests, where $n$ is the number of qualifying alleles. All SNP and indel locations are given in NCBI hg18/Build 36 coordinates unless otherwise specified. Indels are designated as chr:position:I. Multi-allelic variants are designated as chr:position:0:allele, for example, 'chr8:77605042:0:GAA'.

We imputed the genotypes of 4,572 Icelanders with BCC (cases) and 266,358 controls. The BCC cases were comprised of 2,980 individuals who had been directly typed on Illumina SNP genotyping microarray chips and 1,592 close relatives of chip-typed individuals. The controls were comprised of 87,820 chip-genotyped individuals and 178,538 close relatives of chip-typed individuals. For the HumanHap series of chips, 304,937 SNPs were used for long-range phasing, whereas for the Omni series of chips 564,196 SNPs were used. An initial imputation step was carried out on each chip series separately to create a single harmonized, long-range phased genotype data set consisting of 707,525 SNPs. In this first step, sharing between individuals with the same long $(>10 \mathrm{cM})$ haplotypes was used to fill in genotypes of SNPs not present on both chip platforms. So, for example, if a parent was genotyped with a HumanHap series chip and an offspring with an Omni chip, then the alleles of the transmitted chromosome can be inferred for both individuals. Since chip genotypes are available for a large fraction of the Icelandic population (which currently numbers 320,000 ), this process is quite complete with only $1 \%$ of alleles remaining unknown. Subsequently, this genotype data set was used in the second step of imputing the full set of variants which was used in the 24,988,228 tests of association. Association testing was performed using logistic regression, treating disease status as the response and genotype counts as covariates. Other available individual characteristics that correlate with disease status were also included in the model as nuisance variables. These characteristics were gender, county of birth, current age or age at death (first- and second-order terms included), blood sample availability for the individual and an indicator function for the overlap of the lifetime of the individual with the timespan of phenotype collection ${ }^{21}$. Correction for familial relatedness was carried out using genomic control and $P$ values were adjusted accordingly $(\lambda=1.2374)$. Tests for heterogeneity were performed by comparing the null hypothesis of the effect being the same in all populations to the alternative hypothesis of each population having a different effect, using a likelihood ratio test. For conditional analyses, the allele count of each individual was given as a covariate in the logistic regression. All $r^{2}$ values quoted refer to the Icelandic population and were determined using long-range haplotype phasing and imputation.

Assessment for overlap with regulatory regions. To identify a set of BCCassociated variants that might have regulatory effects, we took the index variant at each locus and adjusted its association signal in turn for the effect of each other variant within $\pm 500 \mathrm{~kb}$. If the resulting $P_{\text {adj }}$ was $>0.001$, we considered that the tested variant could not safely be distinguished from the index variant and should therefore be evaluated for potential regulatory and pathogenic effect (irrespective of its $r^{2}$ value relative to the index variant). This resulted in sets containing 45 variants for $2 \mathrm{p} 24 M Y C N$, 41 variants for $8 \mathrm{q} 21$ ZFHX4, 52 variants for 10p14 GATA3 and 195 variants for the 2q33 CASP8-ALS2CR12 locus. Note that for this analysis, we used only variants that were detected by the wholegenome sequencing and which could be imputed with stringent information values $\geq 0.9$ (ref. 15).

For each variant in the sets thus identified, we searched for overlaps with known regulatory regions as follows: first, we used ENSEMBL to determine whether the variant had been assigned a regulatory region ENSR number. Then we examined the ENCODE data and recorded any evidence of ChIP-Seq transcription factor binding, DNaseI hypersensitivity sites, enhancer and promoter chromatin segmentation states ${ }^{59-61}$. We also recorded enhancer and promoter chromatin segmentation states using the 25 state HMM from the Roadmap consortium ${ }^{62}$ Then we looked for correlations between DNaseI hypersensitive sites and local gene expression using results described by Sheffield et al. ${ }^{63}$ We examined SiPhy and GERP conservation scores and the Combined Annotation-Dependent Depletion Scaled C-score ${ }^{25,64,65}$. We also viewed the Factorbook and HaploReg_v2 data ${ }^{66,67}$ (http://www.broadinstitute.org/mammals/haploreg/haploreg.php) to search for potential changes in transcription factor-binding motifs but we did not annotate the variants with these data unless there was ChIP-Seq evidence of the cognate transcription factor binding at that location. To narrow down the variants for scrutiny at each locus, we pass-filtered the variants on (a) being the index BCCassociated marker at the locus or (b) presence of an ENSR number or (c) a reference to normal human keratinocytes (NHEK) or primary foreskin keratinocytes (PFK) in the annotations derived above. After filtration, there remained 2 variants at MYCN, 3 at ZFHX4, 2 at GATA3 and 32 at CASP8ALS2CR12. We manually checked the data for each of these variants in the UCSC GRCh37/hg19 Test Genome Browser and by using HaploReg_v2. The results were tabulated using a style based on the HaploReg_v2 presentation (Supplementary Data 1). We also manually checked the annotations for the unfiltered variants with $P_{\text {adj }}>0.001$ from each locus. This resulted in the identification of two additional variants at the GATA3 locus that are located in GATA3 transcription factorbinding sites but did not meet the pass-filter qualifications (see main text and Supplementary Data 1).

Gene expression microarrays. Samples of RNA from human peripheral blood $(N=1001)$ and adipose tissue $(N=673)$ were hybridized to Agilent Technologies Human $25 \mathrm{~K}$ microarrays ${ }^{26}$. We quantified the expression changes between two samples as the mean logarithm $\left(\log _{10}\right)$ expression ratio (MLR) compared with a reference pool RNA sample. In comparing the expression levels between groups of individuals with different genotypes, we denoted the expression level for each genotype as $10^{\text {(average MLR }}$, where the MLR is averaged over individuals with the particular genotype. We determined the s.e. and significance by regressing the MLR values against the number of risk alleles carried. We took into account the effects of age, gender and differential cell type count in blood as explanatory variables in the regression. $P$ values were adjusted for familial relatedness of the individuals by simulation. No significant eQTLs were associated with the risk alleles at the $2 \mathrm{p} 24$ MYCN, 8q21 ZFHX4 or 10p14 GATA3 loci.

RNA-seq. For preparation of poly-A complementary DNA (cDNA) sequencing libraries, the quality and quantity of isolated total RNA from 261 peripheral blood samples was assessed using the Total RNA 6000 Nano chip for the Agilent 2100 Bioanalyzer. Samples were from different individuals than those who had been sampled for the gene expression microarray studies described above. cDNA libraries derived from Poly-A mRNA were generated using Illumina's TruSeq RNA Sample Prep Kit. In brief, Poly-A mRNA was isolated from total RNA samples (1-4 $\mu \mathrm{g}$ input) using hybridization to Poly-T beads. The Poly-A mRNA was fragmented at $94^{\circ} \mathrm{C}$, and first-strand cDNA was prepared using random hexamers and the SuperScript II reverse transcriptase (Invitrogen). Following second-strand cDNA synthesis, end repair, addition of a single A base, adaptor ligation, AMPure bead purification and PCR amplification, the resulting cDNA was measured on a Bioanalyzer using the DNA 1000 Lab Chip. 
Samples for sequencing were clustered on to flowcells using Illumina's cBot and the TruSeq PE cluster kits v2, respectively. Paired-end sequencing $(2 \times 76$ cycles $)$ was performed with either GAIIx instruments using the TruSeq SBS kits v5 from Illumina or HiSeq 2000 instruments using TruSeq v3 flowcells/SBS kits. In some instances, the read length was $2 \times 50$ or $2 \times 101$ cycles. Approximately, 125-175 million forward reads (250-350M total reads) were sequenced per sample.

RNA sequencing reads were aligned to Homo sapiens Build 36 with TopHat ${ }^{68}$ version 1.4.1 with a supplied set of known transcripts in GTF format (RefSeq hg18; Homo sapiens, NCBI, build36.3). TopHat was configured such that it attempts first to align reads to the provided transcriptome then, for reads that do not map fully to the transcriptome, it attempts to map them onto the genome. Read mapping statistics used for read count normalization were calculated using the CollectRnaSeqMetrics tool in Picard version 1.79 (http://picard.sourceforge.net/ command-line-overview.shtml\#CollectRnaSeqMetrics). Once read alignment by TopHat was finished, gene expression estimation was done using Cufflinks ${ }^{68}$ version 1.3.0 for the same set of transcripts used in the read alignment by TopHat.

For analysis of RNA-seq samples, the normalized read count was determined for each bp location as the number of reads covering the base/the total number of aligned reads in the individual in billions. Then the median normalized read count for each genotype group was determined for each bp location and plotted graphically. To quantify the levels of expression in different regions, the median of normalized read counts over the genomic segment in question was determined for each individual. We then did a logarithmic transformation of the normalized read counts and standardized them so that the response variable had a mean 0 and s.d. of 1 . We determined the effect and significance by regressing the transformed read counts against the number of risk alleles carried. Effect sizes $(\beta)$ are given in s.d. units.

Data from the GTEx Release phs000424.v4.p1 from 17.01.2014 were downloaded from the GTEx consortium ${ }^{35}$ (http://www.gtexportal.org/home/) via dbGAP. Analysis was conducted using the provided aligned read and genotype data as described above for deCODE data. One exception was the integrated CASP8 whole-gene read count by genotype calculation for skin (Supplementary Table 2) which was taken directly from the GTEx portal and employed their methods (see URLs).

RT-PCR. We converted total RNA (from a subset of 76 of the blood RNA samples that were used for the expression microarrays) to cDNA using the High Capacity cDNA Archive Kit (Applied Biosystems), primed with random hexamers. We designed assays spanning the exon-intron junction between exons 8 and 9 (RTPCR common isoforms, Fig. 2, F-primer: $5^{\prime}$-tatatcccggatgaggctga-3', R-primer: $5^{\prime}$ tcaatcagaagggaagacaagtt- $\left.3^{\prime}\right)$, spanning from exon 6 to a sequence unique to exon 8L (RT-PCR 8L splice variant, Fig. 2, F-primer: $5^{\prime}$-gcctgctgaagataatcaacg-3', R-primer: $5^{\prime}$-gaaacatggcccttttggta- $\left.3^{\prime}\right)$ and contained within intron $8,5^{\prime}$ to the NM_033358 probe sequence (RT-PCR retained intron, Fig. 2, F-primer: 5' tcaatttatgaatggacaaccaa- $3^{\prime}$, R-primer: $5^{\prime}$-cctctcttcgtattagagcctttc- $\left.3^{\prime}\right)$. Primer sequences are available on request. We carried out quantitative PCR according to the manufacturer's instructions on an ABI Prism 7900HT Sequence Detection System. We assessed differences in mean relative RNA abundance between genotypic groups by regression, as described above for expression microarrays.

\section{References}

1. Epstein, E. H. Basal cell carcinomas: attack of the hedgehog. Nat. Rev. Cancer 8, 743-754 (2008).

2. Mohan, S. V. \& Chang, A. L. S. Advanced basal cell carcinoma: epidemiology and therapeutic innovations. Curr. Dermatol. Rep. 3, 40-45 (2014).

3. Scherer, D. \& Kumar, R. Genetics of pigmentation in skin cancer-a review. Mutat. Res. 705, 141-153 (2010).

4. Fan, Z. et al. A missense mutation in PTCH2 underlies dominantly inherited NBCCS in a Chinese family. J. Med. Genet. 45, 303-308 (2008).

5. Pastorino, L. et al. Identification of a SUFU germline mutation in a family with Gorlin syndrome. Am. J. Med. Genet. A 149A, 1539-1543 (2009).

6. Smyth, I. et al. Isolation and characterization of human patched 2 (PTCH2), a putative tumour suppressor gene inbasal cell carcinoma and medulloblastoma on chromosome 1p32. Hum. Mol. Genet. 8, 291-297 (1999).

7. Bastiaens, M. T. et al. Melanocortin-1 receptor gene variants determine the risk of nonmelanoma skin cancer independently of fair skin and red hair. Am. J. Hum. Genet. 68, 884-894 (2001).

8. Box, N. F. et al. Melanocortin-1 receptor genotype is a risk factor for basal and squamous cell carcinoma. J. Invest. Dermatol. 116, 224-229 (2001).

9. Gudbjartsson, D. F. et al. ASIP and TYR pigmentation variants associate with cutaneous melanoma and basal cell carcinoma. Nat. Genet. 40, 886-891 (2008).

10. Stacey, S. N. et al. New common variants affecting susceptibility to basal cell carcinoma. Nat. Genet. 41, 909-914 (2009).

11. Nan, H., Kraft, P., Hunter, D. J. \& Han, J. Genetic variants in pigmentation genes, pigmentary phenotypes, and risk of skin cancer in Caucasians. Int. J. Cancer 125, 909-917 (2009).

12. Han, J. et al. A germline variant in the interferon regulatory factor 4 gene as a novel skin cancer risk locus. Cancer Res. 71, 1533-1539 (2011).
13. Praetorius, C. et al. A polymorphism in IRF4 affects human pigmentation through a tyrosinase-dependent MITF/TFAP2A pathway. Cell 155, 1022-1033 (2013).

14. Stacey, S. N. et al. Common variants on $1 \mathrm{p} 36$ and $1 \mathrm{q} 42$ are associated with cutaneous basal cell carcinoma but not with melanoma or pigmentation traits. Nat. Genet. 40, 1313-1318 (2008).

15. Stacey, S. N. et al. A germline variant in the TP53 polyadenylation signal confers cancer susceptibility. Nat. Genet. 43, 1098-1103 (2011).

16. Stacey, S. N. et al. Germline sequence variants in TGM3 and RGS22 confer risk of basal cell carcinoma. Hum. Mol. Genet. 23, 3045-3053 (2014).

17. Rafnar, T. et al. Sequence variants at the TERT-CLPTM1L locus associate with many cancer types. Nat. Genet. 41, 221-227 (2009).

18. Sigurdardottir, L. G. et al. Data quality at the Icelandic Cancer Registry: comparability, validity, timeliness and completeness. Acta Oncol. 51, 880-889 (2012).

19. Kong, A. et al. Detection of sharing by descent, long-range phasing and haplotype imputation. Nat. Genet. 40, 1068-1075 (2008).

20. Kong, A. et al. Parental origin of sequence variants associated with complex diseases. Nature 462, 868-874 (2009).

21. Steinthorsdottir, V. et al. Identification of low-frequency and rare sequence variants associated with elevated or reduced risk of type 2 diabetes. Nat. Genet. 46, 294-298 (2014).

22. Choi, E. \& Cho, C. Expression of a sperm flagellum component encoded by the Als2cr12 gene. Gene Expr. Patterns 11, 327-333 (2011).

23. Van Raam, B. J. \& Salvesen, G. S. Proliferative versus apoptotic functions of caspase- 8 Hetero or homo: the caspase- 8 dimer controls cell fate. Biochim. Biophys. Acta 1824, 113-122 (2012).

24. Jones, E. A., Sajid, M. I., Shenton, A. \& Evans, D. G. Basal cell carcinomas in gorlin syndrome: a review of 202 patients. J. Skin Cancer 2011, 217378 (2011).

25. Kircher, M. et al. A general framework for estimating the relative pathogenicity of human genetic variants. Nat. Genet. 46, 310-315 (2014).

26. Emilsson, V. et al. Genetics of gene expression and its effect on disease. Nature 452, 423-428 (2008).

27. Horiuchi, T. et al. Dominant expression of a novel splice variant of caspase- 8 in human peripheral blood lymphocytes. Biochem. Biophys. Res. Commun. 272, 877-881 (2000).

28. Boldin, M. P., Goncharov, T. M., Goltsev, Y. V. \& Wallach, D. Involvement of $\mathrm{MACH}$, a novel MORT1/FADD-interacting protease, in Fas/APO-1- and TNF receptor-induced cell death. Cell 85, 803-815 (1996).

29. Eckhart, L. et al. Alternative splicing of caspase- 8 mRNA during differentiation of human leukocytes. Biochem. Biophys. Res. Commun. 289, 777-781 (2001).

30. Nagy, E. \& Maquat, L. E. A rule for termination-codon position within introncontaining genes: when nonsense affects RNA abundance. Trends Biochem. Sci. 23, 198-199 (1998).

31. Muzio, M. et al. FLICE, a novel FADD-homologous ICE/CED-3-like protease, is recruited to the CD95 (Fas/APO-1) death-inducing signaling complex. Cell 85, 817-827 (1996).

32. Scaffidi, C., Medema, J. P., Krammer, P. H. \& Peter, M. E. FLICE is predominantly expressed as two functionally active isoforms, caspase-8/a and caspase-8/b. J. Biol. Chem. 272, 26953-26958 (1997).

33. Himeji, D. et al. Characterization of caspase-8L: a novel isoform of caspase- 8 that behaves as an inhibitor of the caspase cascade. Blood 99, 4070-4078 (2002).

34. Mohr, A. et al. Caspase-8L expression protects CD34+ hematopoietic progenitor cells and leukemic cells from CD95-mediated apoptosis. Oncogene 24, 2421-2429 (2005).

35. GTEx Consortium. The genotype-tissue expression (GTEx) project. Nat. Genet. 45, 580-585 (2013).

36. MacPherson, G. et al. Association of a common variant of the CASP 8 gene with reduced risk of breast cancer. J. Natl Cancer Inst. 96, 1866-1869 (2004).

37. Cox, A. et al. A common coding variant in CASP8 is associated with breast cancer risk. Nat. Genet. 39, 352-358 (2007).

38. Barrett, J. H. et al. Genome-wide association study identifies three new melanoma susceptibility loci. Nat. Genet. 43, 1108-1113 (2011).

39. Berndt, S. I. et al. Genome-wide association study identifies multiple risk loci for chronic lymphocytic leukemia. Nat. Genet. 45, 868-876 (2013).

40. Sun, T. et al. A six-nucleotide insertion-deletion polymorphism in the CASP8 promoter is associated with susceptibility to multiple cancers. Nat. Genet. 39, 605-613 (2007).

41. Haiman, C. A. et al. A promoter polymorphism in the CASP8 gene is not associated with cancer risk. Nat. Genet. 40, 259-260 (2008).

42. Lin, W.-Y. et al. Identification and characterisation of novel associations in the CASP8/ALS2CR12 region on chromosome 2 with breast cancer risk. Hum. Mol. Genet. 24, 285-298 (2014).

43. Huang, M. \& Weiss, W. A. Neuroblastoma and MYCN. Cold Spring Harb. Perspect. Med. 3, a014415 (2013).

44. Roussel, M. F. \& Robinson, G. W. Role of MYC in medulloblastoma. Cold Spring Harb. Perspect. Med. 3, a014308 (2013). 
45. Eckhart, L., Lippens, S., Tschachler, E. \& Declercq, W. Cell death by cornification. Biochim. Biophys. Acta 1833, 3471-3480 (2013).

46. Kovalenko, A. et al. Caspase- 8 deficiency in epidermal keratinocytes triggers an inflammatory skin disease. J. Exp. Med. 206, 2161-2177 (2009).

47. Lee, P. et al. Dynamic expression of epidermal caspase 8 simulates a wound healing response. Nature 458, 519-523 (2009).

48. Kaiser r, W. J. et al. RIP3 mediates the embryonic lethality of caspase-8deficient mice. Nature 471, 368-372 (2011)

49. Park, W. S. et al. Inactivating mutations of the caspase-10 gene in gastric cancer. Oncogene 21, 2919-2925 (2002).

50. Shin, M. S. et al. Inactivating mutations of CASP10 gene in non-Hodgkin lymphomas. Blood 99, 4094-4099 (2002).

51. Sanna, M. G. et al. ILPIP, a novel anti-apoptotic protein that enhances XIAPmediated activation of JNK1 and protection against apoptosis. J. Biol. Chem. 277, 30454-30462 (2002).

52. Chou, J., Provot, S. \& Werb, Z. GATA3 in development and cancer differentiation: cells GATA have it! J. Cell. Physiol. 222, $42-49$ (2010).

53. Kurek, D., Garinis, G. A., van Doorninck, J. H., van der Wees, J. \& Grosveld, F. G. Transcriptome and phenotypic analysis reveals Gata3-dependent signalling pathways in murine hair follicles. Development 134, 261-272 (2007).

54. Kaufman, C. K. et al. GATA-3: an unexpected regulator of cell lineage determination in skin. Genes Dev. 17, 2108-2122 (2003).

55. Rácz, E. et al. GATA3 expression is decreased in psoriasis and during epidermal regeneration; induction by narrow-band UVB and IL-4. PLOS ONE 6, e19806 (2011).

56. De Guzman Strong, C. et al. Lipid defect underlies selective skin barrier impairment of an epidermal-specific deletion of Gata-3. J. Cell Biol. 175, 661-670 (2006).

57. Ouyang, W. et al. Stat6-independent GATA-3 autoactivation directs IL-4independent Th2 development and commitment. Immunity 12, 27-37 (2000).

58. Tjønneland, A. et al. Study design, exposure variables, and socioeconomic determinants of participation in Diet, Cancer and Health: a population-based prospective cohort study of 57,053 men and women in Denmark. Scand. J. Public Health 35, 432-441 (2007).

59. Dunham, I. et al. An integrated encyclopedia of DNA elements in the human genome. Nature 489, 57-74 (2012).

60. ENCODE. A user's guide to the encyclopedia of DNA elements (ENCODE). PLoS Biol. 9, e1001046 (2011).

61. Ernst, J. et al. Mapping and analysis of chromatin state dynamics in nine human cell types. Nature 473, 43-49 (2011).

62. Chadwick, L. H. The NIH Roadmap Epigenomics Program data resource. Epigenomics 4, 317-324 (2012).

63. Sheffield, N. C. et al. Patterns of regulatory activity across diverse human cell types predict tissue identity, transcription factor binding, and long-range interactions. Genome Res. 23, 777-788 (2013).

64. Davydov, E. V. et al. Identifying a high fraction of the human genome to be under selective constraint using GERP ++ . PLoS Comput. Biol. 6, e1001025 (2010).

65. Garber, M. et al. Identifying novel constrained elements by exploiting biased substitution patterns. Bioinformatics 25, i54-i62 (2009).

66. Wang, J. et al. Sequence features and chromatin structure around the genomic regions bound by 119 human transcription factors. Genome Res. 22, 1798-1812 (2012).

67. Ward, L. D. \& Kellis, M. HaploReg: a resource for exploring chromatin states, conservation, and regulatory motif alterations within sets of genetically linked variants. Nucleic Acids Res. 40, D930-D934 (2011).

68. Trapnell, C. et al. Differential gene and transcript expression analysis of RNA-seq experiments with TopHat and Cufflinks. Nat. Protoc. 7, 562-578 (2012).

\section{Acknowledgements}

We thank G.H. Olafsdottir of the Icelandic Cancer Registry for assistance in the ascertainment of affected individuals, Eirikur Steingrimsson for helpful comments on the manuscript and Hjalti Sigurdsson for help with the graphics. In Valencia, Spain the samples from the Instituto Valenciano de Oncología were retrieved from the Bionbank of the Instituto Valenciano de Oncología. In Copenhagen, Denmark the study 'Diet, Cancer and Health' was supported by grants from the Danish Cancer Society and 'Europe Against Cancer': European Prospective Investigation into Cancer and Nutrition (EPIC). We are grateful to the GTEx Consortium for early stage sharing of RNA-seq and genotypic data. The following acknowledgement refers to the GTEx data: the Genotype-Tissue Expression (GTEx) Project was supported by the Common Fund of the Office of the Director of the National Institutes of Health. Additional funds were provided by the NCI, NHGRI, NHLBI, NIDA, NIMH and NINDS. Donors were enrolled at Biospecimen Source Sites funded by NCI $\backslash$ SAIC-Frederick, Inc. (SAIC-F) subcontracts to the National Disease Research Interchange (10XS170), Roswell Park Cancer Institute (10XS171) and Science Care Inc. (X10S172). The Laboratory, Data Analysis and Coordinating Center (LDACC) was funded through a contract (HHSN268201000029C) to The Broad Institute Inc. Biorepository operations were funded through an SAIC-F subcontract to Van Andel Institute (10ST1035). Additional data repository and project management were provided by SAIC-F (HHSN261200800001E). The Brain Bank was supported by a supplements to University of Miami grants DA006227 \& DA033684 and to contract N01MH000028. Statistical Methods development grants were made to the University of Geneva (MH090941 \& MH101814), the University of Chicago (MH090951, MH090937, MH101820, MH101825), the University of North Carolina-Chapel Hill (MH090936 \& MH101819), Harvard University (MH090948), Stanford University (MH101782), Washington University St Louis (MH101810) and the University of Pennsylvania (MH101822).

\section{Author contributions}

S.N.S., H.H., S.A.G., G.T., F.Z., P.S., A.K., D.F.G., U.T., J.H.O. and K.S. designed the study and interpreted the results. S.N.S., B.S., K.R.B., K.T., R.R., V.F., C.C., Y.G., M.G., D.P., O.S., P.R., E.G., K.K., B.A.N., A.T., K.O., J.G.J., L.T., T.R., U.V., R.K., E.N., J.I.M. and J.H.O carried out the subject ascertainment and recruitment. S.N.S., H.H., G.T., F.Z., A.S., H.J., A.M.K., H.S., G.M., O.T.M., T.R. and U.T. performed the sequencing, genotyping and expression analyses. S.N.S., H.H., S.A.G., G.T., F.Z., A.S., B.K., P.S., G.M., B.V.H., A.K. and D.F.G. performed the statistical and bioinformatics analyses. S.N.S., H.H., S.A.G., G.T., F.Z. and K.S. drafted the manuscript. All authors contributed to the final version of the paper. Principle collaborators for the case-control population samples were B.S. and J.H.O. (Iceland), U.V. (Denmark), R.K. (Eastern Europe), E.N. (Valencia, Spain) and J.I.M (Zaragoza, Spain)

\section{Additional information}

Supplementary Information accompanies this paper at http://www.nature.com/ naturecommunications

Competing financial interests: All deCODE authors are employees of the biotechnology firm deCODE Genetics, a subsidiary of AMGEN.

Reprints and permission information is available online at http://npg.nature.com/ reprintsandpermissions/

How to cite this article: Stacey, S. N. et al. New basal cell carcinoma susceptibility loci. Nat. Commun. 6:6825 doi: 10.1038/ncomms7825 (2015).

(c) (i) This work is licensed under a Creative Commons Attribution 4.0 International License. The images or other third party material in this article are included in the article's Creative Commons license, unless indicated otherwise in the credit line; if the material is not included under the Creative Commons license, users will need to obtain permission from the license holder to reproduce the material. To view a copy of this license, visit http://creativecommons.org/licenses/by/4.0/ 